\title{
THE PROBABILITY OF EVENTUAL RUIN IN THE COMPOUND BINOMIAL MODEL
}

\author{
By Elias S. W. SHIU \\ University of Manitoba, Canada
}

\begin{abstract}
This paper derives several formulas for the probability of eventual ruin in a discrete-time model. In this model, the number of claims process is assumed to be binomial. The claim amounts, premium rate and initial surplus are assumed to be integer-valued.
\end{abstract}

\section{KEYWORDS}

Compound binomial process; Probability of eventual ruin; Ultimate ruin probability; Infinite-time ruin probability; Risk theory; Random walk; Gambler's ruin; Lagrange series.

\section{INTRODUCTION AND NOTATION}

This paper is motivated by the recent paper GERBER (1988b), which discusses the probability of eventual ruin in a discrete-time model. We shall derive some of GERBER'S results by alternative methods. As we shall point out below, our formulation and notation are not exactly the same as GERBER'S.

We consider a discrete-time model, in which the number of insurance claims is governed by a binomial process $N(t), t=0,1,2, \ldots$ In any time period, the probability of a claim is $q$ (denoted by $p$ in GerBer's paper) and the probability of no claim is $1-q$. The occurrences of a claim in different time periods are independent events. The individual claim amounts $X_{1}, X_{2}, X_{3}, \ldots$ are mutually independent, identically distributed, positive and integer-valued random variables; they are independent of the binomial process $N(t)$. Put $X=X_{1}$, and let $p(x)=\operatorname{Pr}(X=x)$. The value of the probability density function $p(x)$ is zero unless $x$ is a positive integer. We also assume that the premium received in each period is one and is larger than the net premium $q E(X)$. Put $E(X)=\mu$; then the last assumption is

$$
1>q \mu \text {. }
$$

For $k=1,2,3, \ldots$, define

$$
S_{k}=X_{1}+X_{2}+\ldots+X_{k} .
$$

ASTIN BULLETIN, Vol. 19, No. 2 
Put $S_{0}=0$. Let the initial risk reserve be a nonnegative integral amount $u$. The probability of eventual ruin (ultimate ruin probability, infinite-time ruin probability) $\psi(u)$ is the probability that the risk reserve

$$
U(t)=u+t-S_{N(t)}
$$

is ever negative. Since Gerber (1988b) defines ruin as the event that the risk reserve $U(t)$ becomes nonpositive for some $t, t>0$, the formulas derived below will not be exactly the same as his.

\section{THE PROBABILITY OF NONRUIN}

It is somewhat easier to work with the nonruin function

$$
\phi(u)=1-\psi(u) .
$$

For $u<0, \phi(u)=0$. Consider an initial risk reserve of amount $j, j \geq 0$. If there is no claim in the first period, the risk reserve becomes $j+1$ at the end of the period; if there is a claim of amount $x$ in the first period, the risk reserve becomes $j+1-x$. Hence, by the law of total probability,

$$
\phi(j)=(1-q) \phi(j+1)+q E[\phi(j+1-X)], \quad j=0,1,2, \ldots
$$

Rearranging (2.1) yields

$$
\phi(j+1)-\phi(j)=q\{\phi(j+1)-E[\phi(j+1-X)]\}, \quad j=0,1,2, \ldots
$$

Summing (2.2) from $j=0$ to $j=k-1$, we have

$$
\phi(k)-\phi(0)=q\left\{\sum_{j=1}^{k} \phi(j)-E\left[\sum_{j=1}^{k} \phi(j-X)\right]\right\}, k=1,2,3, \ldots,
$$

or

$$
\phi(k)-(1-q) \phi(0)=q\left\{\sum_{j=0}^{k} \phi(j)-E\left[\sum_{j=1}^{k} \phi(j-X)\right]\right\}, k=1,2,3, \ldots
$$

Let $1+$ denote the function defined by

$$
\begin{array}{ll}
1_{+}(j)=1, & j=0,1,2, \ldots, \\
1_{+}(j)=0, & j=-1,-2, \ldots .
\end{array}
$$

For each pair of functions $f$ and $g$, let $f * g$ denote their convolution,

$$
(f * g)(j)=\sum_{i=-\infty}^{\infty} f(j-i) g(i) \text {. }
$$

Note that, if $f(i)=g(i)=0$ for all negative integers $i$, then (2.4) becomes

$$
(f * g)(j)=\sum_{i=0}^{j} f(j-i) g(i) .
$$


Since the convolution operation can be regarded as a multiplication operation between functions, we sometimes write $(f * g)(j)$ as $f(j) * g(j)$.

The first sum in the right-hand side of $(2.3)$ is $\left(\phi * 1_{+}\right)(k)$. As $X$ is a positive random variable,

$$
\sum_{j=1}^{k} \phi(j-X)=\sum_{j=0}^{k} \phi(j-X)=\left(\phi * 1_{+}\right)(k-X) .
$$

Hence, (2.3) becomes

$$
\begin{aligned}
\phi(k)-(1-q) \phi(0) & =q\left\{\left(\phi * 1_{+}\right)(k)-E\left[\left(\phi * 1_{+}\right)(k-X)\right]\right\} \\
& =q\left[\left(\phi * 1_{+}\right)(k)-\left(\phi * 1_{+} * p\right)(k)\right], \quad k=1,2,3, \ldots .
\end{aligned}
$$

Since $p(0)=0$, it is easy to check that (2.6) also holds for $k=0$. To solve for $\phi$ in (2.6), we first extend it as an equation for all integers $k$, positive and negative:

$$
\phi(k)-(1-q) \phi(0) 1_{+}(k)=q\left[\left(\phi * 1_{+}\right)(k)-\left(\phi * 1_{+} * p\right)(k)\right] .
$$

Let $\delta$ be the function defined by $\delta(0)=1$ and $\delta(j)=0$ for $j \neq 0$. Then the right-hand side of (2.7) can be expressed as

$$
q\left\{\phi(k) * 1_{+}(k) *[\delta(k)-p(k)]\right\} .
$$

Rearranging (2.7) and writing

$$
c=(1-q) \phi(0)
$$

yields

$$
\phi(k) *\left(\delta(k)-q\left\{1_{+}(k) *[\delta(k)-p(k)]\right\}\right)=c 1_{+}(k) .
$$

Equation (2.9) is a Volterra equation of the second kind. To solve for $\phi$, we invert

$$
\delta(k)-q\left\{I_{+}(k) *[\delta(k)-p(k)]\right\}
$$

as the Neumann series [BROWN and PAGE (1970, p. 226), RIESZ and Sz.-NAGY (1955, p. 146)]

$$
\sum_{n=0}^{\infty} q^{n}\left\{1_{+}(k) *[\delta(k)-p(k)]\right\}^{* n}
$$

(We use the notation: $f^{* 0}=\delta$ and $f^{* n}=f^{*(n-1)} * f, n=1,2,3, \ldots$ ). Hence,

$$
\phi(k)=c \sum_{n=0}^{\infty} q^{n}\left\{[\delta(k)-p(k)]^{* n} * 1 *_{+}^{(n+1)}(k)\right\}
$$


Since

$$
\begin{aligned}
& {[\delta(k)-p(k)]^{* n} }=\sum_{j=0}^{n}\left(\begin{array}{l}
n \\
j
\end{array}\right)(-1)^{j} p^{* j}(k), \\
& 1_{+}^{*(n+1)}(k)=\left(\begin{array}{c}
k+n \\
n
\end{array}\right) 1_{+}(k), \\
&\left(\begin{array}{c}
n \\
j
\end{array}\right)\left(\begin{array}{c}
k+n \\
n
\end{array}\right)=\left(\begin{array}{c}
k+j \\
j
\end{array}\right)\left(\begin{array}{c}
k+n \\
n-j
\end{array}\right), \\
& \sum_{n=j}^{\infty}\left(\begin{array}{c}
k+n \\
n-j
\end{array}\right) q^{n-j}=\left(\frac{1}{1-q}\right)^{k+j+1}
\end{aligned}
$$

and

$$
p^{* j}(k) * f(k)=E\left[f\left(k-S_{j}\right)\right],
$$

by an interchange of the order of summation (2.11) becomes

$$
\begin{aligned}
\phi(k) & =c \sum_{j=0}^{\infty}(-q)^{j}\left\{p^{* j}(k) *\left[\left(\begin{array}{c}
k+j \\
j
\end{array}\right)\left(\frac{1}{1-q}\right)^{k+j+1} 1_{+}(k)\right]\right\} \\
& =\phi(0) \sum_{j=0}^{\infty}\left(\frac{-q}{1-q}\right)^{j} E\left[\left(\begin{array}{c}
k+j-S_{j} \\
j
\end{array}\right)(1-q)^{S_{j}-k} 1_{+}\left(k-S_{j}\right)\right]
\end{aligned}
$$

As $S_{j} \geq j$, there are at most $k+1$ nonzero terms in the right-hand side of (2.13). This formula corresponds to (4.6) of $\mathrm{SHIU}_{\mathrm{H}}$ (1988) and (3.14) of SHIU (1989a).

To derive the value of $\phi(0)$, we return to formula (2.6). Let $P$ denote the probability distribution function of the individual claim amount random variable $X$. Then

$$
P=1_{+} * p
$$

As $k$ tends to positive infinity, the left-hand side of (2.6) tends to

$$
1-(1-q) \phi(0)
$$

while the right-hand side tends to

$$
\begin{aligned}
q \sum_{j=-\infty}^{\infty}[1+(j)-P(j)] & =q \sum_{j=0}^{\infty}[1-P(j)] \\
& =q \mu
\end{aligned}
$$


by the Lebesgue dominated convergence theorem. Hence.

$$
\phi(0)=\frac{1-q \mu}{1-q} .
$$

\section{GAMBLER'S RUIN}

As a verification of formulas (2.13) and (2.14), let us consider the special case that $X \equiv 2$. This is a classical problem in the theory of random walk. The probability that, with an initial reserve of $u$ (a nonnegative integer), the company's risk reserve will ever become -1 is known to be $[q /(1-q)]^{u+1}$.

Since $S_{j}=2 j$, formula (2.13) becomes

$$
\begin{aligned}
\phi(u) & =\frac{\phi(0)}{(1-q)^{u}} \sum_{j=0}^{\infty}[-q(1-q)]^{j}\left(\begin{array}{c}
u-j \\
j
\end{array}\right) 1_{+}(u-2 j) \\
& =\frac{1-2 q}{(1-q)^{u+1}} \sum_{j=0}^{\llbracket u / 2 \rrbracket}[-q(1-q)]^{j}\left(\begin{array}{c}
u-j \\
j
\end{array}\right) .
\end{aligned}
$$

For a real number $r$, we let $\llbracket r \rrbracket$ denote the greatest integer less than or equal to $r$. The polynomial

$$
\sum_{n=0}^{[k / 2 \rrbracket}\left(\begin{array}{c}
k-n \\
n
\end{array}\right) x^{n}
$$

is related to the Chebyshev polynomials of the second kind and can be expressed as [KNUTH (1973, problem 1.2.9.15), RIORDAN (1968, p. 76)]

$$
\frac{(1+\sqrt{1+4 x})^{k+1}-(1-\sqrt{1+4 x})^{k+1}}{2^{k+1} \sqrt{1+4 x}} .
$$

Now,

$$
\begin{aligned}
\sqrt{1-4 q(1-q)} & =|2 q-1| \\
& =1-2 q
\end{aligned}
$$

by assumption (1.1). Hence,

$$
\phi(u)=1-\left(\frac{q}{1-q}\right)^{u+1}
$$

as required.

For the case that $X \equiv m>2$, formula (2.13) cannot be simplified. It has been given by Burman (1946). Also see Girshick (1946, p. 290), SeAl (1962, p. 23; 1969, p. 101) and Gerber (1988b, (43)). 


\section{ANOTHER RUIN PROBABILITY FORMULA}

GERBER (1988b) has derived another formula for the probability of eventual ruin, which is complementary to (2.13). It follows from condition (1.1) that

$$
\operatorname{Pr}\left[\lim _{t \rightarrow \infty} U(t)=+\infty\right]=1 \text {. }
$$

If ruin occurs, there is necessarily a last upcrossing of the risk reserve $U(t)$ from level -1 to level 0 . By considering the number of claims $n$, prior to this last upcrossing, and the time $t$ at which it occurs, we have

(4.1) $\psi(u)=\left[\sum_{n=1}^{\infty} \sum_{t=n}^{\infty}\left(\begin{array}{l}t \\ n\end{array}\right) q^{n}(1-q)^{t-n} \operatorname{Pr}\left(S_{n}=u+t+1\right)\right](1-q) \phi(0)$.

Since

$$
\begin{aligned}
& \sum_{t=n}^{\infty}\left(\begin{array}{l}
t \\
n
\end{array}\right)(1-q)^{t} \operatorname{Pr}\left(S_{n}=u+t+1\right) \\
& =E\left[\left(\begin{array}{c}
S_{n}-u-1 \\
n
\end{array}\right)(1-q)^{S_{n}-u-1} 1_{+}\left(S_{n}-u-n-1\right)\right],
\end{aligned}
$$

we obtain the formula

$$
\begin{aligned}
\psi(u)= & (1-q \mu) \sum_{n=1}^{\infty}\left(\frac{q}{1-q}\right)^{n} \\
& E\left[\left(\begin{array}{c}
S_{n}-u-1 \\
n
\end{array}\right)(1-q)^{S_{n}-u-1} 1_{+}\left(S_{n}-u-n-1\right)\right] .
\end{aligned}
$$

Continuous-time analogues of (4.2) can be found in PraBHU (1965, (5.55)), Gerber (1988a, (27)) and Shiu (1989a, (1.6)).

\section{GERBER'S FANCY SERIES}

Using the identity

$$
(-1)^{j}\left(\begin{array}{c}
-a \\
j
\end{array}\right)=\left(\begin{array}{c}
a+j-1 \\
j
\end{array}\right)
$$

we can rewrite (2.13) as

(5.1) $\phi(u)=(1-q \mu) \sum_{j=0}^{\infty}\left(\frac{q}{1-q}\right)^{j} E\left[\left(\begin{array}{c}S_{j}-u-1 \\ j\end{array}\right)(1-q)^{S_{j}-u-1} 1_{+}\left(u-S_{j}\right)\right]$. 
Since

$$
\phi(u)+\psi(u)=1
$$

and $u$ is an integer, adding (5.1) to (4.2) yields

$$
\frac{1}{1-q \mu}=\sum_{n=0}^{\infty}\left(\frac{q}{1-q}\right)^{n} E\left[\left(\begin{array}{c}
S_{n}+x \\
n
\end{array}\right)(1-q)^{S_{n}+x}\right] \text {, }
$$

if we put $x=-(u+1)$. This interesting formula is Theorem la of GERBER (1988b). In this section we present some alternative proofs for (5.2); the assumption that $x$ is an integer will not be used.

Assume that all the moments of the random variable $X$ exist. Consider the linear operator $G$ on the linear space of polynomials defined by

$$
(G f)(y)=E[f(y+X)] .
$$

[Such operators have been considered by FelLeR (1971, section VIII.3)]. As $f$ is a polynomial, the random variable $f(y+X)$ in (5.3) can be expressed as

$$
\sum_{j \geq 0} \frac{X^{j} f^{(j)}(y)}{j !} .
$$

Consequently, the linear operator $G$ can be represented as a power series in terms of the differentiation operator $D$ :

$$
G=\sum_{j \geq 0} \frac{E\left(X^{j}\right)}{j !} D^{j}
$$

Since

$$
G-I=\mu D+1 / 2 E\left(X^{2}\right) D^{2}+\ldots,
$$

we have, for each nonnegative integer $n$,

$$
(G-I)^{n} x^{n}=n ! \mu^{n}
$$

and, for nonnegative integers $n$ and $m, m<n$,

$$
(G-I)^{n} x^{m}=0 .
$$

It follows from (5.6) and (5.7) that

$$
(G-I)^{n}\left(\begin{array}{l}
x \\
n
\end{array}\right)=\mu^{n}
$$

Multiplying (5.8) with $q^{n}$ and summing from $n=0$ and $n=\infty$ yields 


$$
\sum_{n=0}^{\infty} q^{n}(G-I)^{n}\left(\begin{array}{l}
x \\
n
\end{array}\right)=\frac{1}{1-q \mu} .
$$

Applying the formulas

$$
\begin{aligned}
& (G-I)^{n}=\sum_{k=0}^{n}\left(\begin{array}{l}
n \\
k
\end{array}\right)(-1)^{n-k} G^{k} \\
& \left(\begin{array}{l}
x \\
n
\end{array}\right)\left(\begin{array}{l}
n \\
k
\end{array}\right)=\left(\begin{array}{l}
x \\
k
\end{array}\right)\left(\begin{array}{l}
x-k \\
n-k
\end{array}\right)
\end{aligned}
$$

and

$$
\sum_{n=k}^{\infty}(-1)^{n-k}\left(\begin{array}{c}
x-k \\
n-k
\end{array}\right) q^{n-k}=(1-q)^{x-k}
$$

we obtain

$$
\sum_{k=0}^{\infty}\left(\frac{q}{1-q}\right)^{k} G^{k}\left[\left(\begin{array}{l}
x \\
k
\end{array}\right)(1-q)^{x}\right]=\frac{1}{1-q \mu}
$$

Since

$$
\left(G^{k} f\right)(x)=E\left[f\left(x+S_{k}\right)\right], \quad k=0,1,2, \ldots,
$$

formula (5.10) is the same as (5.2).

An operational calculus proof is (5.10) can be found in SHIU (1989b).

If the random variable $X$ in formula (5.2) is degenerate, i.e., $X \equiv \mu$, then we have

$$
\sum_{n=0}^{\infty}\left(\begin{array}{c}
x+\mu n \\
n
\end{array}\right)\left[q(1-q)^{\mu-1}\right]^{n}=\frac{1}{(1-\mu q)(1-q)^{x}}
$$

This result is quite well known; it and its variants can be found in Póly (1922, (7)), WhitTaker and WATSON (1927, p. 133, example 3), RIORDAN (1968, p. 147), Pólya and Szegö (1970, p. 126, problem 216), KNUTH (1973, problem 1.2.6.26), Melzak (1973, p. 117, example 4), Comtet (1974, p. 153), Henricl (1974, p. 121, problem 12), Rota (1975, p. 56), Roman and Rota (1978, p. 115) and HofRi (1987, p. 34). The standard proof of formula (5.11) is by an application of the Lagrange series formula. The proof can readily be generalized to one for (5.2), as we shall show below. (Also see section 5 of SHIU (1989a)).

Let $h$ be an analytic function and let

$$
z=b+w h(z)
$$


By the implicit function theorem, there is a unique root $z=z(w)$ which reduces to $b$ at $w=0$. If $f$ is an analytic function, then $f(z)=f(z(w))$ may be expressed as follows [RIORdan (1968, p. 146), PÓLYA and SzEGO (1970, p. 125), GOULDEN and JACKSON (1983, p. 17)]:

$$
\frac{f(z)}{1-w h^{\prime}(z)}=\sum_{j=0}^{\infty} \frac{w^{j}}{j !}\left[\frac{d^{j}}{d y^{j}}\left[f(y)[h(y)]^{j}\right]\right]_{y=b} .
$$

Now, consider $b=1-q$,

$$
f(y)=y^{x}
$$

and

$$
h(y)=E\left(y^{x}\right)
$$

Then

$$
[h(y)]^{j}=E\left(y^{s_{j}}\right)
$$

and

$$
\frac{1}{j !} \frac{d^{j}}{d y^{j}}\left[f(y)[h(y)]^{j}\right]=E\left[\left(\begin{array}{c}
S_{j}+x \\
j
\end{array}\right) y^{S_{\jmath}+x-j}\right] .
$$

With $w=q$, the right-hand side of (5.13) is the same as the right-hand side of (5.2) and equation (5.12) becomes

$$
z=(1-q)+q E\left(z^{X}\right) .
$$

Thus $z=1$ and the left-hand side of (5.13) is identical to the left-hand side of (5.2).

\section{REMARKS}

(i) Consider formula (2.14). Since $X \geq 1$ by hypothesis, the number $\phi(0)$ is always bounded above by one as it should be. If $1 \leq q \mu$, then ruin is guaranteed; but this is ruled out by condition (1.1). It follows from (2.14) that

$$
\psi(0)=\frac{q(\mu-1)}{1-q} .
$$

However, Gerber's (1988b) result is that

$$
\psi(0)=q \mu .
$$

This discrepancy exists because GeRBER defines ruin to occur when the risk reserve $U(t)$ becomes nonpositive, while we consider the insurance company to 
be solvent even if its risk reserve is zero. An anonymous referee has kindly pointed out that our definition of ruin is equivalent to DUFRESNE's (1988, section 3) and (2.14) is DufresNe's formula (37).

(ii) Gerber (1988b) first obtained formula (5.2) and then derived a formula corresponding to (4.1). With these two formulas, he derived formulas corresponding to (2.14) and (2.13).

(iii) Formula (2.12) is a special case of the combinatorial identity

$$
\sum_{k=0}^{r}\left(\begin{array}{c}
r-k \\
m
\end{array}\right)\left(\begin{array}{c}
s+k \\
n
\end{array}\right)=\left(\begin{array}{c}
r+s+1 \\
m+n+1
\end{array}\right)
$$

where $m, n, r$ and $s$ are nonnegative integers and $n \geq s$ [RIORDAN $(1968, \mathrm{p} .35$, problem 13), KNUTH (1973, p. 58), HofRI (1987, p. 39, problem 2b)].

(iv) Formula (2.1) can written as

(6.2) $\phi(j+1)-\phi(j)=[q /(1-q)]\{\phi(j)-E[\phi(j+1-X)]\}, \quad j=0,1,2, \ldots$

Hence, for each positive integer $k$,

$$
\begin{aligned}
\phi(k)-\phi(0) & =[q /(1-q)]\{\phi(k)-E[\phi(k+1-X)]\} * 1_{+}(k-1) \\
& =[q /(1-q)]\left\{\phi(k) *\left[1_{+}(k-1)-P(k)\right]\right\},
\end{aligned}
$$

which is reminiscent of a renewal equation in the compound Poisson model [(Feller, 1971, (XI.7.2)), (SHIU, 1989a, (2.4))]. Let $h$ denote the function

$$
h(k)=\left[1_{+}(k-1)-P(k)\right] /(\mu-1), \quad k=0, \pm 1, \pm 2, \ldots .
$$

It follows from (6.3) and (6.1) that, for all integers $k$,

$$
\phi(k)-\phi(0) 1_{+}(k)=\psi(0)[\phi(k) * h(k)] .
$$

Define $H^{* n}=h^{* n} * 1_{+}$. Then

$$
\phi(u)=\phi(0) \sum_{n=0}^{\infty}[\psi(0)]^{n} H^{* \prime \prime}(u)
$$

Formula (6.4) is analogous to a convolution series formula in the compound Poisson model; see SHIU $(1988,(2.1) ; 1989 \mathrm{a},(2.14))$. Since $h(i)=0$ for all $i \leq 0$, there are at most $u+1$ nonzero terms in the right-hand side of (6.4), i.e.,

$$
\phi(u)=\phi(0) \sum_{n=0}^{u}[\psi(0)]^{n} H^{* n}(u)
$$

As

$$
\sum_{n=0}^{\infty}[\psi(0)]^{n}=1 /[1-\psi(0)]=1 / \phi(0),
$$


we have, for each nonnegative integer $u$,

$$
\psi(u)=[1-\psi(0)] \sum_{n=1}^{\infty}[\psi(0)]^{n}\left[1-H^{* n}(u)\right] .
$$

Formula (6.6) has been derived by R. MICHEL and can be found in a forthcoming risk theory book by C. HIPP and R. MICHEL. Observe that, when $X \equiv 2, h(j)=\delta(j-1)$ and formula (3.4) immediately follows from (6.5). I thank $C$. Hipp for the information above.

\section{ACKNOWLEDGMENT}

Support from the Natural Sciences and Engineering Research Council of Canada is gratefully acknowledged.

\section{REFERENCES}

BURman, J. P. (1946) Sequential sampling formulac for a binomial population. Journal of the Royal Statistical Society B 8, 98-103.

Brown, A.L. and PAGE, A. (1970) Elements of Fumctional Analysis. Van Nostrand Reinhold, London.

Comtet, L. (1974) Advanced Combinatorics: The Art of Finite and Infinite Expansions. Reidel, Dordrecht.

DUFRESNE, F. (1988) Distributions stationnaires d'un système bonus-malus et probabilité et ruine. ASTIN Bulletin 18, 31-46.

Feller, W. (1971) An Introduction to Probability Theory and Its Applications, Vol, 2 (2nd edn). Wiley, New York.

Gerber, H. U. (1988a) Mathematical fun with ruin theory. Insurance: Mathematics and Economics 7, 15-23.

GERBER, H. U. (1988b) Mathematical fun with the compound binomial process. ASTIN Bulletin 18, $16 !-168$.

Girshick, M.A. (1946) Contributions to the theory of sequential analysis, II, III. Anmals of Mathematical Statistics 17, 282-298.

Henrici, P. (1974) Applied and Compunational Complex Analysis, Vol. 1 : Power Series - Integration - Conformal Mapping - Location of Zeros. Wiley, New York.

Hofri, M. (1987) Probabilistic Analysis of Algorithms: On Computing Methodologies for Computer Algorithms Performance Evaluation. Springer-Verlag, New York.

KNUTH, D. E. (1973) The Art of Computer Programming, Vol. 1: Fundamental Algorithms (2nd edn). Addison-Wesley, Reading, Massachusetts.

Melzak, Z. A. (1973) Companion to Concrete Marhematics: Mathematical Techniques and Various Applications. Wiley, New York.

POLYA, G. (1922) Sur les séries entières dont la soinme est une fonction algébrique. L'Enseignement Mathématique 22, 38-47. Reprinted in George Pólya: Collected Papers, Vol. 1: Singularities of Analytic Functions. MIT Press, Cambridge, Massachusselts (1974), pp. 165-174.

Polya, G. and Szego, G. (1970) Aufgabe und Lehrsäze aus der Analysis. Vol. I (4th edn). Springer-Verlag, Berlin.

PRABHU, N. U. (1965) Queues and Inventories: A Siudy of Their Basic Siochastic Processes. Wiley, New York.

RIESZ, F. and SZ.-NAGY, B. (1955) Functional Analysis. Ungar, New York. An English translation of Lecons d'analyse fonctionelle (deuxième ćdition), Akadèmiai Kiadó, Budapest (1953).

RIORDAN, J. (1968) Combinatorial Identities. Wiley, New York. Reprinted with correction by Kricger, Huntington, New York (1979),

Roman, S.M. and Rota, G.-C. (1978) The umbral calculus. Advances in Mathematics 27, 95-188. 
Rota, G.-C. (1975) Finite Operator Calculus. Academic Press, New York.

SEAL, H. L. (1962) The random walk of a simple risk business. ASTIN Bulletin 4, 19-28.

SEAL, H. L. (1969) Siochastic Theory of a Risk Business. Wiley, New York.

SHIU, E.S.W. (1988) Calculation of the probability of eventual ruin by Beekman's convolution series. Insurance: Mathematics and Economics 7, 41-47.

SHIU, E.S.W. (1989a) Ruin probability by operational calculus. Insurance: Mathematics and Economics, forthcoming.

SHIU, E. S. W. (1989b) On Gerber's fun. Scandinavian Actuarial Journal, forthcoming.

Whittaker, E.T. and Watson, G. N. (1927) A Course of Modern Analysis (4th edn). Cambridge University Press, London.

Elias S. W. Shiu

Department of Actuarial and Management Sciences, University of Manitoba, Winnipeg, Manitoba R3T 2N2, Canada. 